\title{
LXXXV.-On the heat of vapours and on astronomical refractions
}

\author{
John William Lubbock Esq. Treas. R.S. F.R.A.S. F.L.S.
}

To cite this article: John William Lubbock Esq. Treas. R.S. F.R.A.S. F.L.S. (1840) LXXXV.-On the heat of vapours and on astronomical refractions , Philosophical Magazine Series 3, 16:106, 562-569, DOI: $10.1080 / 14786444008650093$

To link to this article: http://dx.doi.org/10.1080/14786444008650093

册 Published online: 01 Jun 2009.

Submit your article to this journal $\widetilde{x}$

Џll Article views: 2

Q View related articles $\asymp$ 
appears to open a field for discovery, which experimental industry cannot fail to cultivate with immediate success.

Note.-On the evening of the meeting at which my investigations were presented to the Society, my friend, Dr. Bache of the Girard College, gave an account of the investigations of Professor Ettingshausen of Vienna, in reference to the improvement of the magneto-electric machine, some of the results of which he had witnessed at the University of Vienna about a year since. No published account of these experiments has yet reached this country, but it appears that Professor Ettingshausen had been led to suspect the development of a current in the metal of the keeper of the magnetoelectric machine, which diminished the effect of the current in the coil about the keeper, and hence to separate the coil from the keeper by a ring of wood of some thickness, and afterwards, to prevent entirely the circulation of currents in the keeper, by 'dividing it into segments, and separating them by a non-conducting material. I am not aware of the result of this last device, nor whether the mechanical difficulties in its execution were fully overcome. It gives me pleasure to learn that the improvements, which I have merely suggested as deductions from the principles of the interference of induced currents (76), should be in accordance with the experimental conclusions of the above-named philosopher.

LXXXV.-On the Heat of Vapours and on Astronomical Refractions. By John William Lubbock, Esq., Treas. R.S. F.R.A.S and F.L.S., Vice-Chancellor of the University of London, \&c.

[Continued from p. 514.]

ON THE STEAM-ENGINE.

THE law which connects the pressure and the temperature of steam having been unknown, various empirical rules have been given. As, however, the expressions which arise are not in a convenient form for the calculations which are required in order to ascertain the duty which steam-engines are capable of performing, or to solve other problems of the same nature, M. de Pambour*, in his work on that subject, has employed another expression, viz.

$$
\mu=\frac{1}{\rho}=\frac{1}{n+q p},
$$

in which $\varrho$ is the density of steam, $p$ the pressure, and $n$ and $q$ constants. According to my expression

* Théorie de la Machine à Vapeur, p. 111. 


$$
\frac{1}{\varrho}=\frac{(1-E)}{p^{\frac{1}{\gamma}}-E p}
$$

The pressure being reckoned in atmospheres, and the density of steam corresponding to the pressure of one atmosphere (or 14.706 lbs. per square inch) being unity. If we take the density of water for unity, then as the volume of steam at the pressure of one atmosphere is 1700 times greater than that of the same weight of water,

$$
\begin{aligned}
\mu & =-\frac{K}{\rho^{\frac{1}{\gamma_{*}}}-E p} \quad K=1700(E-1) \\
\log K=2 \cdot 4765041 & \frac{1}{\gamma}=1 \cdot 0134 \quad E=1 \cdot 17602 .
\end{aligned}
$$

If we suppose that a certain volume of water represented by $S$ be transformed into vapour at the pressure $p$, and that $M$ is the absolute volume of vapour which results, we shall have

$$
\frac{M}{\bar{S}}=\mu=\frac{[0 \cdot 4109002]}{p}\left(\frac{1}{\alpha}+\theta\right) .
$$

If afterwards the same volume of water is transformed into vapour at the pressure $p^{\prime}$, and that the absolute volume which the resulting vapour occupies be called $M^{\prime}$, we shall have

$$
\begin{aligned}
& \frac{M^{\prime}}{S}=\mu^{\prime} \\
& \frac{M}{M^{\prime}}=\frac{p^{\prime} \frac{1}{\gamma}-E p}{p^{\frac{1}{\gamma}}-E p}
\end{aligned}
$$

"Soit* $\boldsymbol{P}$ la pression totale de la vapeur dans la chaudière, et $p^{\prime}$ la pression qu'aura cette vapeur à son arrivée dans le cylindre, pression qui sera toujours moindre que $P$, excepté dans un cas particulier que nous traiterons plus loin. La vapeur pénétrera donc dans le cylindre à la pression $p^{\prime}$, et elle continuera d'affluer avec cette pression et de produire un effet correspondant, jusqu'à ce que la communication entre la chaudière et le cylindre soit interceptée. Alors il cessera d'arriver de la vapeur nouvelle dans le cylindre, mais celle qui y est déjà parvenue, commencera à se dilater pendant le reste de la course du piston, en produisant par sa détente une certaine quantité de travail, qui s’ajoutera à celle déjà produite pendant la période d'admission de la vapeur.

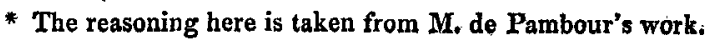


" $P$ étant la pression de la vapeur dans la chaudic̀re, et $p^{\prime}$ la pression qu'elle prendra à son arrivée dans le cylindre avant la détente, soit $\pi$ la pression de cette vapeur en un point quelconque de la détente. Soit en même temps $l$ la longueur totale de la course $\mathrm{du}$ piston, $l^{\prime}$ la portion parcourue au moment où a commencé la détente, et $\lambda$ celle qui correspond au point où la vapeur a acquis la pression $\pi$. Enfin, soit encore $a$ l'aire du piston, et $c$ la liberté du cylindre, c'est-à-dire l'espace libre qui existe à chaque bout du cylindre, au-delà de la portion parcourue par le piston, et qui se remplit nécessairement de vapeur à chaque course; cet espace, y compris les passages aboutissants, étant représenté par une longueur équivalente du cylindre.

"Si l'on prend le piston au moment où la longueur de course parcourue est $\lambda$, et la pression $\pi$, on verra que si le piston parcourt, en outre, un espace élémentaire $d \lambda$, le travail élémentaire produit dans ce mouvement sera $\pi a \mathrm{~d} \lambda$. Mais en même temps, le volume $a\left(l^{\prime}+c\right)$ occupé par le vapeur avant la détente sera devenu $a(\lambda+c) . "$ Hence,

$$
\begin{gathered}
\frac{M}{\bar{M}^{\prime}}=\frac{p^{\prime \frac{1}{\gamma}}-E p^{\prime}}{\pi^{\frac{1}{\gamma}}-E \pi}=\frac{\lambda+c}{l^{\prime}+c} . \\
\lambda+c=\left(l^{\prime}+c\right) \frac{\left(p^{\frac{1}{\gamma}}-E p^{\prime}\right)}{\left(\pi^{\frac{1}{\gamma}}-E \pi\right)}
\end{gathered}
$$

The elementary work produced $=\pi a \mathrm{~d} \lambda$.

$$
\begin{aligned}
& \int \pi a \mathrm{~d} \lambda=\pi a(\lambda+c)-\int a(\lambda+c) \mathrm{d} \pi \\
&=\pi a(\lambda+c)-\int a\left(l^{\prime}+c\right)\left(p^{\prime \frac{1}{\gamma}}-E p^{\prime}\right) \frac{\mathrm{d} \sigma}{\left(\pi^{\frac{1}{\gamma}}-E \pi\right)} \\
&=\pi a(\lambda+c)-a\left(l^{\prime}+c\right)\left(p^{\prime} \frac{1}{\gamma}-E p^{\prime}\right) \int \frac{\mathrm{d} \sigma}{\pi^{\frac{1}{\gamma}}-E \pi} \\
&=\pi a(\lambda+c) \\
&+a\left(l^{\prime}+c\right) \frac{\left(p^{\frac{1}{\gamma}}-E p^{\prime}\right) \gamma}{E(\gamma-1)} \log \left(1-E \pi^{\frac{\gamma-1}{\gamma}}\right)+\text { const. }
\end{aligned}
$$

This integral is to be taken from $\lambda=l^{\prime}$ to $\lambda^{\prime}=l$, let $\pi=p$ when $\lambda=l$, when $\lambda=l^{\prime}, \pi=p^{\prime}$. 


$$
\begin{gathered}
\int \pi a \mathrm{~d} \lambda=p a(l+c)-p^{\prime} a\left(l^{\prime}+c\right) \\
+a\left(l^{\prime}+c\right) \frac{\left(p^{\frac{1}{\gamma}}-E p^{\prime}\right) \gamma}{E(\gamma-1)} \log \left\{\frac{1-E p^{\frac{\gamma-1}{\gamma}}}{1-E p^{\frac{\gamma-1}{\gamma}}}\right\}
\end{gathered}
$$

for the values of the constants $E, \gamma$. See p. 511 .

To this must be added the work effected during the course of the piston through $l^{\prime}$, which is $p^{\prime} a l$, and if $R$ is the total pressure exerted upon unity of surface of the piston

$p a(l+c)-p^{\prime} a c$

$$
\begin{aligned}
&+a\left(l^{\prime}+c\right)\left(p^{\frac{1}{\gamma}}-E p^{\prime}\right) \frac{\gamma}{E(\gamma-1)} \log \left\{\frac{1-E p^{\frac{\gamma-1}{\gamma}}}{1-E p^{\frac{\gamma-1}{\gamma}}}\right\} \\
&=a R l
\end{aligned}
$$

$R=(1+\delta) r+p^{\prime \prime}+f, f$ is the friction of the machine not loaded, $\delta$ the increase of this friction due to unity of the charge $r, p^{\prime \prime}$ the pressure on the surface of the piston, representing the atmospheric pressure when the machine works without condensation, and otherwise the pressure of condensation in the cylinder.

If $S$ denote the volume of water converted into vapour by the boiler in unity of time, this volume in the cylinder becomes

$$
-\frac{S K}{p^{\frac{1}{\gamma}}-E p^{\prime}}
$$

$K$ being the same constant as in p. 563 .

It is evident, according to the reasoning of M. de Pambour, in p. 125. of his work, that if $v$ denote the velocity of the piston

$$
\frac{S K}{\frac{1}{p^{\prime} r}-E p^{\prime}}=-v a \frac{l^{\prime}+c}{l}
$$

This equation is equivalent to the equation (A) of M. de Pambour, p. 123, which may be put into the form

or

$$
\begin{aligned}
& p a(l+c)-p^{\prime} a c+\frac{l s}{q^{v}} \text { Nap. } \log \left\{\frac{l+c}{l^{\prime}+c}\right\}=a R l . \\
& p a(l+c) \cdots p^{\prime} a c+[6.9505960] \frac{l S}{v} \log \left\{\frac{l+c}{l^{\prime}+c}\right\}=a R h
\end{aligned}
$$

the pressure being reckoned in lbs. per square inch. 


$$
p^{\prime} \frac{1}{\gamma}-E p^{\prime}=-\frac{l S K}{a v\left(l^{\prime}+c\right)}=\frac{K}{\mu^{\prime}},
$$

Similarly,

$$
\begin{aligned}
& p^{\frac{1}{\gamma}}-E p=-\frac{l S K}{a v(l+c)}=\frac{K}{\mu} \\
& \frac{1}{\mu}=\frac{l S}{a v(l+c)} \quad \frac{1}{\mu^{\prime}}=\frac{l S}{a v\left(l^{\prime}+c\right)} \\
& p a(l+c)-p^{\prime} a c \\
& -\frac{l S K \gamma}{v E(1-\gamma)} \text { Nap.log }\left\{\frac{1-E p^{\frac{\gamma-1}{\gamma}}}{1-E p^{\frac{\gamma-1}{\gamma}}}\right\}=a R l \\
& p a(l+c)-p^{\prime} a c-\left(p^{\prime \prime}+f\right) a l \\
& -[4.6411966] \frac{l S}{v} \log *\left\{\frac{1-E p^{\frac{\gamma-1}{\gamma}}}{1-E p^{\frac{\gamma-1}{\gamma}}}\right\}=a l(1+\delta) r \\
& \log \left\{\frac{1-E p^{\frac{\gamma-1}{\gamma}}}{1-E p^{\frac{\gamma-1}{\gamma}}}\right\}=\log \left\{\frac{l+c}{l^{\prime}+c}\right\}-\frac{1}{\gamma} \log \left(\frac{p^{\prime}}{p}\right) \text {. }
\end{aligned}
$$

If the machine work without expansion,

$$
\begin{aligned}
& p=p^{\prime}, \quad \log \left\{\frac{1-E p^{\frac{\gamma-1}{\gamma}}}{1-E p^{\frac{\gamma-1}{\gamma}}}\right\}=0, \\
& r=\frac{p-\left(p^{\prime \prime}+f\right)}{1+\delta}
\end{aligned}
$$

The data upon questions relating to the steam-engine are the quantities $a, l, l^{\prime}, S$, and $v$, and it is evident that from these quantities the quantities $\mu$ and $\mu^{\prime}$ may at once be found by an easy arithmetical operation; from these the following table will give the corresponding pressures $p$ and $p^{\prime}$, and these pressures being introduced into equation $A$, the value of $a r$ may be easily found.

* Log. of Briggs, the pressure being reckoned in atmospheres, the log. of the constant is $[7.9670537]$, the pressure being reckoned in lbs. per square foot. 
Table showing the volume (compared with that of water at $212^{\circ}$ ), and the temperature of steam.

\begin{tabular}{|c|c|c|c|c|c|c|c|}
\hline \multirow{2}{*}{$\begin{array}{c}\text { Pressure } \\
\text { in lbs. per } \\
\text { square inch. } \\
14.706 \times p \text {. }\end{array}$} & \multicolumn{2}{|c|}{$\begin{array}{c}\text { Temperature. } \\
\text { Fahrenheit. } \\
\boldsymbol{\tau}\end{array}$} & \multirow{2}{*}{$\begin{array}{l}\text { Volume. } \\
\qquad \mu \uparrow\end{array}$} & \multirow{2}{*}{$\begin{array}{l}\text { Pressure } \\
\text { in lbs. per } \\
\text { square inch. } \\
14: 706 \times p \text {. }\end{array}$} & \multicolumn{2}{|c|}{$\begin{array}{c}\text { Temperature. } \\
\text { Fahrenheit. } \\
\boldsymbol{T} \\
\end{array}$} & \multirow{2}{*}{$\underset{\mu}{\text { Volume. }}$} \\
\hline & $\begin{array}{l}\text { Air*. } \\
\text { Therm. }\end{array}$ & $\begin{array}{l}\text { Merc. } \\
\text { Therm. }\end{array}$ & & & $\begin{array}{l}\text { Air. } \\
\text { Therm. }\end{array}$ & $\begin{array}{l}\text { Merc. } \\
\text { Therm. }\end{array}$ & \\
\hline 1 & $101 \%$ & $101 \div 5$ & 20816 & 56 & $287^{\circ} \cdot 6$ & 289.5 & 498 \\
\hline 2 & $126 \cdot 0$ & $126^{\circ} 0$ & 10871 & 57 & $288 \cdot 7$ & $290 \cdot 7$ & 490 \\
\hline 3 & $141 \cdot 4$ & $141 \cdot 4$ & 7442 & 58 & $289 \cdot 8$ & $291 \cdot 8$ & 482 \\
\hline 4 & 153.0 & $153 \cdot 0$ & 5691 & 59 & 290.9 & $293 \cdot 0$ & 474 \\
\hline 5 & $162 \cdot 2$ & $162 \cdot 2$ & 4622 & 60 & $292 \cdot 0$ & $294 \cdot 1$ & 467 \\
\hline 6 & $170 \cdot 1$ & $170 \cdot 1$ & 3902 & 61 & $293 \cdot 0$ & $295 \cdot 1$ & 460 \\
\hline 7 & $176 \cdot 8$ & 176.8 & 3381 & 62 & $294 \cdot 0$ & $296 \cdot 2$ & 453 \\
\hline 8 & $182 \cdot 8$ & $182 \cdot 8$ & 2986 & 63 & $295 \cdot 0$ & $297 \cdot 2$ & 447 \\
\hline 9 & $188 \cdot 3$ & $188 \cdot 3$ & 2678 & 64 & $296 \cdot 0$ & $298 \cdot 2$ & 440 \\
\hline 10 & $193 \cdot 2$ & $193 \cdot 2$ & 2429 & 65 & $297 \cdot 0$ & $299 \cdot 2$ & 434 \\
\hline 11 & $197 \cdot 8$ & $197 \cdot 8$ & 2223 & 66 & $298^{\circ} 0$ & $300 \cdot 3$ & 428 \\
\hline 12 & $202 \cdot 0$ & $202 \cdot 0$ & 2051 & 67 & $299 \cdot 0$ & 301.3 & 422 \\
\hline 13 & $205 \cdot 9$ & 205.9 & 1905 & 68 & $300^{\circ} 0$ & 302.3 & 417 \\
\hline 14 & $209 \cdot 5$ & $209 \cdot 5$ & 1778 & 69 & $301 \cdot 0$ & $303 \cdot 3$ & 411 \\
\hline $14 \cdot 706$ & $212 \cdot 0$ & $212 \cdot 0$ & 1700 & 70 & $301 \cdot 9$ & $304 \cdot 2$ & 406 \\
\hline 15 & $212 \cdot 9$ & $212 \cdot 9$ & 1669 & 71 & $302 \cdot 8$ & $305 \cdot 2$ & 401 \\
\hline 16 & 216.3 & $216^{*} 4$ & 1572 & 72 & $303 \cdot 7$ & $306 \cdot 1$ & 396 \\
\hline 17 & $219 \cdot 3$ & $219 \cdot 5$ & 1487 & 73 & $304^{\circ} 6$ & $307 \cdot 0$ & 391 \\
\hline 18 & $222 \cdot 3$ & $222 \cdot 6$ & 1410 & 74 & 305.5 & $308 \cdot 0$ & 386 \\
\hline 19 & $225 \cdot 1$ & $\mathbf{2 2 5} 5$ & 1342 & 75 & $306^{\circ} 4$ & 308.9 & 381 \\
\hline 20 & $227 \cdot 9$ & $228 \cdot 3$ & 1280 & 76 & $307 \cdot 3$ & 309.8 & 377 \\
\hline 21 & $230 \cdot 4$ & $230 \cdot 9$ & 1224 & 77 & $308 \cdot 2$ & $310 \cdot 7$ & 372 \\
\hline 22 & $232 \cdot 9$ & 2335 & 1172 & 78 & $309 \cdot \mathbf{I}$ & $311 \cdot 6$ & 368 \\
\hline 23 & $235 \cdot 2$ & $235 \cdot 8$ & 1125 & 79 & 3100 & $312 \cdot 6$ & 364 \\
\hline 24 & $237 \cdot 6$ & $238 \cdot 3$ & 1082 & 80 & $310 \cdot 9$ & 3135 & 359 \\
\hline 25 & 239.8 & $240 \cdot 6$ & 1042 & 81 & 311.8 & $314 \cdot 5$ & 355 \\
\hline 26 & $242 \cdot 0$ & $242 \cdot 8$ & 1005 & 82 & 312.7 & $315 \cdot 4$ & 351 \\
\hline 27 & 2440 & $\mathbf{2 4 4} \cdot 9$ & 971 & 83 & 3135 & $316^{\circ} 3$ & 348 \\
\hline 28 & $246 \cdot 1$ & $247 \cdot 0$ & 939 & 84 & $\mathbf{3 1 4} \mathbf{3}$ & $317 \cdot 1$ & 344 \\
\hline 29 & 248.0 & $249 \cdot 0$ & 909 & 85 & $315 \cdot 1$ & 317.9 & 340 \\
\hline 30 & $250^{\circ} 0$ & $251 \cdot 0$ & 881 & 86 & $315 \cdot 9$ & 318.7 & 337 \\
\hline 31 & $251 \cdot 8$ & $252 \cdot 8$ & 855 & 87 & 316.7 & $319 \cdot 6$ & 333 \\
\hline 32 & 253.7 & 254.8 & 831 & 88 & $317 \cdot 5$ & $320 \cdot 4$ & 330 . \\
\hline 33 & $255 \cdot 4$ & $256 \cdot 5$ & 808 & 89 & $318 \cdot 3$ & $321 \cdot 2$ & 326 \\
\hline 34 & $257 \cdot 2$ & $258: 3$ & 786 & 90 & $319 \cdot 1$ & $322 \cdot 0$ & 323 \\
\hline 35 & 258.9 & $260 \cdot 1$ & 765 & 91 & $319 \cdot 9$ & 322.9 & 320 \\
\hline 36 & $260^{\circ} 6$ & $261 \cdot 8$ & 746 & 92 & $320 \cdot 7$ & $323 \cdot 7$ & 317 \\
\hline 37 & $262 \cdot 2$ & 263.5 & 727 & 93 & $321 \cdot 5$ & $324 \cdot 5$ & 313 \\
\hline 38 & $263^{\circ} 8$ & $265 \cdot 1$ & 709 & 94 & $\mathbf{3 2 2 \cdot 3}$ & $325 \cdot 3$ & 310 \\
\hline 39 & 265.3 & $266^{\circ} 6$ & 693 & 95 & $323 \cdot 0$ & 3260 & 307 \\
\hline 40 & $266^{\circ} 8$ & $268 \cdot 2$ & 677 & 96 & $323 \cdot 7$ & 326.8 & 305 \\
\hline 41 & $268 \cdot 2$ & $269 \cdot 6$ & 662 & 97 & $324 \cdot 4$ & $327 \cdot 5$ & 302 \\
\hline 42 & $269 \cdot 7$ & $\mathbf{2 7 1 \cdot 2}$ & 647 & 98 & $325 \cdot 1$ & $328 \cdot 3$ & 299 \\
\hline 43 & $271 \cdot 1$ & $272 \cdot 6$ & 633 & 99 & $325 \cdot 8$ & $329 \cdot 0$ & 296 \\
\hline 44 & $272 \cdot 5$ & $274 \cdot 1$ & 620 & 100 & 326.5 & $329 \cdot 7$ & 293 \\
\hline 45 & $273 \cdot 8$ & $275 \cdot 4$ & 608 & 105 & $330 \cdot 0$ & $333 \cdot 3$ & 281 \\
\hline 46 & $275 \cdot 2$ & $276 \cdot 8$ & 596 & 120 & $339 \cdot 7$ & $343 \cdot 4$ & 249 \\
\hline 47 & $\mathbf{8 7 6 . 5}$ & $278 \cdot 2$ & 584 & 135 & $348 \cdot 4$ & $352 \cdot 4$ & 223 \\
\hline 48 & $277 \cdot 8$ & $279 \cdot 5$ & 573 & 150 & 356.5 & $360 \cdot 8$ & 203 \\
\hline 49 & $279 \cdot 1$ & $280 \cdot 8$ & 562 & 165 & $363 \cdot 9$ & $368 \cdot 4$ & 186 \\
\hline 50 & $280 \cdot 4$ & $282 \cdot 1$ & 552 & 180 & $370 \cdot 7$ & $375 \cdot 5$ & 172 \\
\hline 51 & $281 \cdot 7$ & $283 \cdot 5$ & 542 & 195 & $377 \cdot 0$ & $382 \cdot 0$ & 160 \\
\hline 52 & $282 \cdot 9$ & 2847 & 532 & 210 & 383.3 & $388 \cdot 4$ & 150 \\
\hline 53 & $284 \cdot 1$ & $286^{\circ} 0$ & 523 & 225 & $389 \cdot 0$ & $394 \cdot 4$ & 141 \\
\hline 54 & $285 \cdot 2$ & $287 \cdot 1$ & 514 & 240 & 394.5 & $400 \cdot 1$ & 133 \\
\hline 55 & $286^{\circ} 4$ & $288 \cdot 3$ & 506 & & & & \\
\hline
\end{tabular}


The following example will serve to show in what manner the table was calculated.

Ex.-Calculation of the temperature and volume of steam for the pressure of $180 \mathrm{lbs}$. per square inch.

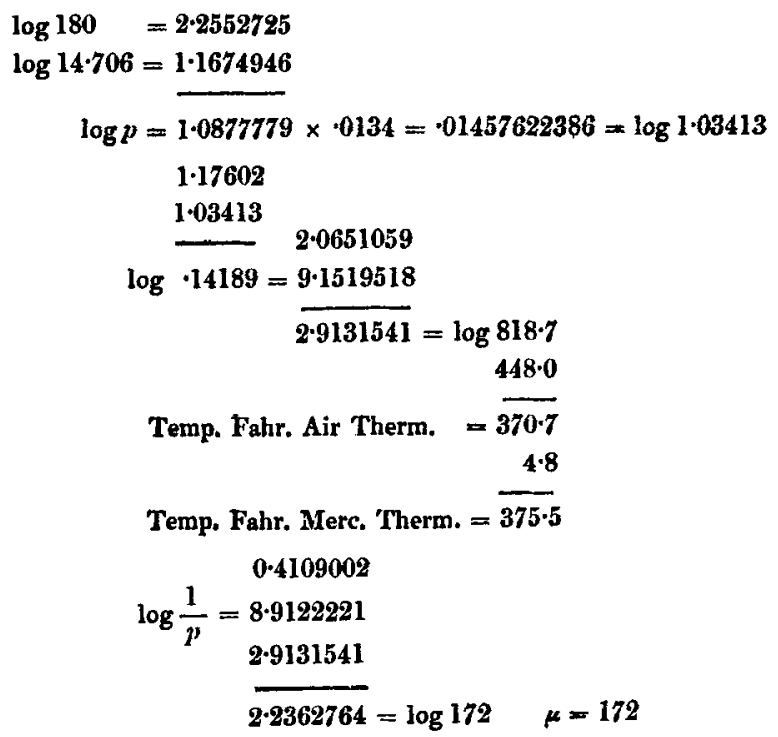

The following data are taken from M. de Pambour's work on the Steam Engine, p. 238.

$$
\begin{aligned}
& l^{\prime}=\cdot 25 l \quad v=250 \quad \mathrm{f}=\cdot 5 \text { (lb. per square inch) } \quad \delta=\cdot 14 \\
& l=10 \mathrm{ft} . \quad \quad \quad \quad \quad S=12.566 \text { sq. ft. } \quad .927 \text { cub. ft. } \\
& \boldsymbol{p}^{\prime \prime}=4 \text { lbs. per square inch. } \quad c=\cdot 05 l \\
& \log v=2 \cdot 3979400 \quad \log \quad v=2 \cdot 3979400 \\
& \log a=1.0991971 \quad \log a=1.0991971 \\
& \log \cdot 30=9 \cdot 4771213 \quad \log 1.05=0.0211893 \\
& \overline{\mathbf{2 \cdot 9 7 4 2 5 8 4}} \overline{\mathbf{3 . 5 1 8 3 2 6 4}} \\
& \log S=9.9670797 \quad \log \quad S=\mathbf{9 . 9 6 7 0 7 9 7} \\
& \overline{3 \cdot 0071787}=\log 1016 \cdot 6 \quad \overline{3.5512467}=\log 3558.3 \\
& \mu^{\prime}=1016 \cdot 6 \quad \mu=3558 \cdot 3
\end{aligned}
$$


Hence by the table $p^{\prime}=25 \cdot 686, p=6.627$ reckoned in lbs. per square inch.

$$
\begin{aligned}
& p^{\prime}=1.7466, \quad p=\cdot 4506, \quad p^{\prime \prime}+f=\cdot 3060 \text { in atmosphleres. } \\
& \log 1 \cdot 7466=\cdot 2422019, \quad \log \cdot 4506=9 \cdot 6538224 \\
& \cdot 2422019 \times \cdot 0134=\cdot 00324550546 \\
& 9.9967545 \\
& \log E=0 \cdot 0704184 \\
& \overline{0 \cdot 0671729}=\log 1 \cdot 16727 \\
& 0 \cdot 3461776 \times \cdot 0134=\cdot 00463877984 \\
& .0704184 \\
& \cdot 0750571=\log 1 \cdot 18865 \\
& \log \cdot 18865=9 \cdot 2756568 \\
& \log \cdot 16727=\mathbf{9} \cdot \mathbf{2 2 3 4 1 8 1} \\
& \overline{0.0522387}
\end{aligned}
$$

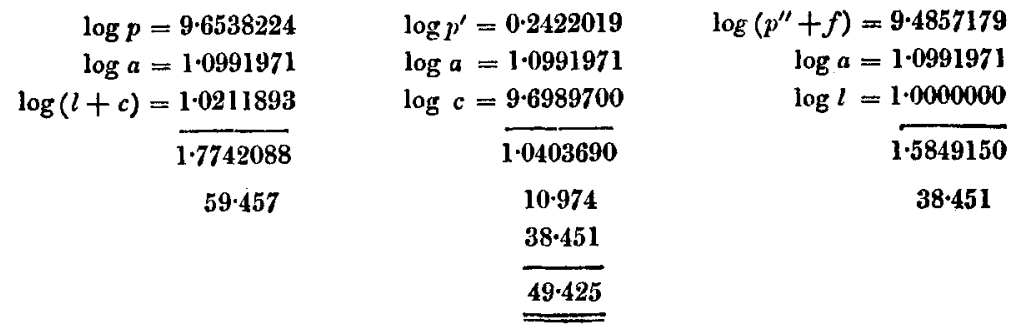

$$
\begin{aligned}
& 4 \cdot 6411966 \\
& \log l=1 \cdot 0000000 \\
& \log S=\mathbf{9 \cdot 9 6 7 1 5 5 4} \\
& \text { 8.7179923 } \\
& 4 \cdot 3263443 \\
& \log v=2 \cdot 3979400 \\
& 1.9284043 \\
& \log 94.834=1.9769641 \\
& \log 14 \cdot 706=1 \cdot 1674946 \\
& \log 144=2 \cdot 1583625 \\
& \overline{5 \cdot 3028212} \\
& \log l(1+\delta)=1 \cdot 0569049 \\
& 4 \cdot 2459163 \\
& 84 \cdot 802 \\
& a r=17616 \\
& 59 \cdot 457 \\
& 144 \cdot 259 \\
& \mathbf{4 9 \cdot 4 2 5}
\end{aligned}
$$

a $r=17616$ expressed in lbs., M. de Pambour finc่s $a r=17337$.

[To be continued.]

Phil. Mag. S. 3. Vol. 16. No. 106. Suppl. July 1840. $2 Q$ 